$58-61$

\title{
Intraoperative neuromonitoring: lessons learned from 32 case events in 2095 spine cases
}

\author{
Authors Matthew Eager, Faisal Jahangiri, Adam Shimer, Francis Shen, Vincent Arlet \\ Institution University of Virginia Health System, Department of Orthopaedic Surgery, Division of Spine Surgery, \\ Charlottesville, Virginia, USA
}

\section{ABSTRACT}

Study type: Restrospective chart review

Introduction: Intraoperative neuromonitoring is becoming the standard of care for many more spinal surgeries, especially with deformity correction and instrumentation. We reviewed our institution's neuromonitored spine cases over the past 4 years to see the immediate intraoperative and postoperative clinical findings when an intraoperative neuromonitoring event was noted.

Objective: The main question addressed in this review is how multimodality intraoperative neuromonitoring has affected our ability to avoid potential neurological injury during spine surgery.

Methods: We retrospectively reviewed 2,095 neuromonitored spine cases at one institution performed over a period of 4 years. Data from the single neuromonitoring provider (Impulse Monitoring, Inc.) at our institution was collected and any cases with possible intraoperative events were isolated. The intraoperative and immediate postoperative clinical documentation of these 32 cases were reviewed (Table 1).

Results: There were 17 cases where changes noted on EMG, SSEP, and/ or MEPs affected the course of the surgery, and prevented possible postoperative neurological deficits. Of these 17, five were related to hypotension, seven due to deformity correction, one screw had a low triggered EMG threshold and was repositioned, and four cases had changes related to patient positioning and external pressure (ie, brachial plexus stretch). None of the 17 cases had postoperative motor or sensory deficits (Figure 1).

No funding was provided to perform this study. This study has been IRB approved. 
Four cases consisted of intradural cord biopsies or tumor resections that had various positive neuromonitoring findings that essentially serve as controls. These cases confirm that the expected changes were seen on neuromonitoring. Four cases had false-positive neuromonitoring findings due to one technical issue requiring needle repositioning, one low threshold with triggered EMG without a pedicle breach, one case had decreased MEP responses with stable SSEPs, and one case had decreased SSEPs after positioning the patient prone. None of these four cases had any postoperative deficits. Four cases showed improved SSEPs after decompression; three cervical corpectomies, and one thoracic discectomy.

Three cases of lumbar instrumentation with spontaneous EMGs each had a medial screw breach without intraoperative findings (Figure 2). They all had a postoperative motor deficit (foot drop). None of these three cases had triggered EMGs performed with the index procedure.

Conclusions: Overall, this review reinforces the importance of multimodality neuromonitoring for spinal surgery. The incidence of possible events in our series was $1.5 \%$. It is difficult to determine the true incidence, since it is impossible to know of any missed events due to lack of complete documentation. In a majority of the cases with events, possible postoperative neurologic deficits were avoided by intraoperative intervention, but the possible outcomes without intervention are not known. Clearly, in the three cases with lumbar pedicle screw malposition, triggered EMGs would have likely shown low thresholds. This would allow for screw reposition, and thus avoid a postoperative lumbar radiculopathy and revision surgery. The incidence of false-positive findings was very low in this review, and unfortunately the true incidence of false-negative findings is not able to be elucidated with this database.
Figure 1 During the insertion of the convex rod: decrease of the MEP amplitude in left foot by $80 \%$ amplitude (yellow arrow). The baseline recording is in blue, the current recording in purple. The right side (non represented) will remain normal.

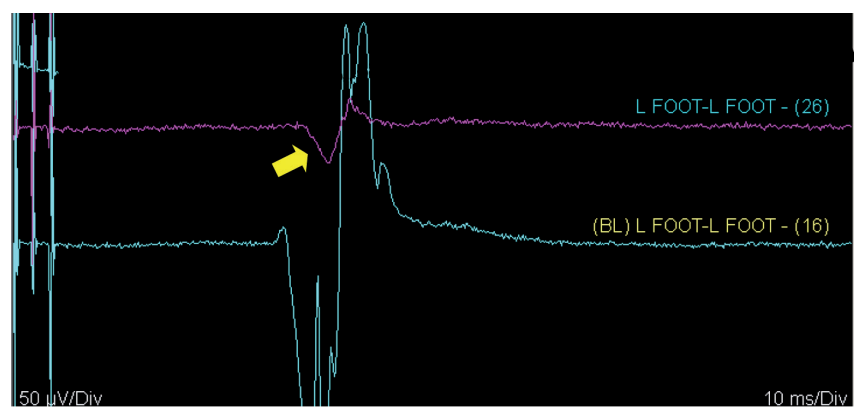

Figure 2 Left L4 pedicle screw medial breach. Triggered EMGs were not performed during the index procedure. Postoperative foot drop required a second surgery to reposition the screw.

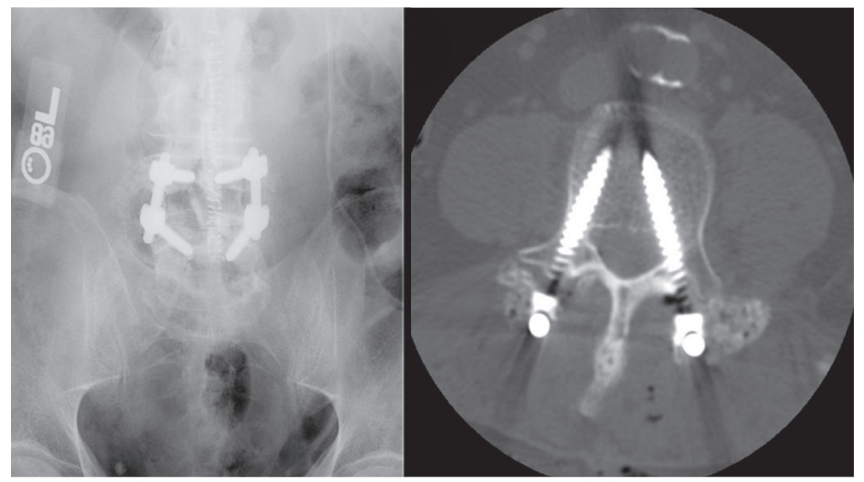




\section{Table 1 Summary of each case event with the type of procedure, intraoperative findings, intraoperative interven-} tion, and postoperative findings

\begin{tabular}{|c|c|c|c|c|}
\hline Case & Procedure & Intraoperative findings & Intraoperative intervention & Postoperative findings \\
\hline 1 & Posterior cervical decopression & Loss of MEP & Increased blood preasure & No deficit \\
\hline 2 & Cervicothoracic spinal cord lesion biopsy & Loss of MEP in lower extremities & None & Bilateral lower extremity paresis \\
\hline 3 & Thoracic spinal cord tumor debulking & Loss of MEP in right lower extremity & None & Right lower extremity paralysis \\
\hline 4 & $\begin{array}{l}\text { Posterior lumbosacral } \\
\text { decompression/fusion TLIF }\end{array}$ & Low S1 screw threshold & Screw checked, repositioned & No deficit \\
\hline 5 & Posterior lumbar decompression/fusion & None & None & Foot drop, medial L4 screw breach \\
\hline 6 & $\begin{array}{l}\text { Cervicomedullary spinal cord } \\
\text { tumor resection }\end{array}$ & Loss of left upper extremity SSEP & None & Left upper extremity sensory deficit \\
\hline 7 & $\begin{array}{l}\text { Anterior thoracic } \\
\text { discectomies/partial corpectomies }\end{array}$ & Left upper extremity decreased SSEP & Carm pressing on arm, removed & No deficit \\
\hline 8 & $\begin{array}{l}\text { Posterior lumbosacral } \\
\text { decompression/fusion TLIF }\end{array}$ & Right upper extremity decreased SSEP & Arm repositioned & No deficit \\
\hline 9 & C7-T1 anterior decompression/fusion & Right lower extremity decreased MEP & Increased blood preasure & No deficit \\
\hline 10 & T11-L5 anterior discectomy/fusion & $\begin{array}{l}\text { Right lower extremity decreased } \\
\text { SSEP, MEP }\end{array}$ & Increased blood preasure & No deficit \\
\hline 11 & $\begin{array}{l}\text { Posterior thoracolumbar } \\
\text { decompression/fusion TLIF }\end{array}$ & Left lower extremity decreased SSEP & Increased blood preasure & No deficit \\
\hline 12 & $\begin{array}{l}\text { Posterior thoracolumbar } \\
\text { decompression/fusion costotransversec- } \\
\text { tomy } T 11, T 12, \mathrm{~L} 1 \text { partial vertebrectomies }\end{array}$ & $\begin{array}{l}\text { Right lower extremity decreased } \\
\text { SSEP, MEP }\end{array}$ & Stopped procedure, stage 1 of 2 & No deficit \\
\hline 13 & $\begin{array}{l}\text { Posterior thoracolumbar } \\
\text { decompression/fusion TLIF }\end{array}$ & Variable SSEP, MEP & Labile blood preasure & No deficit \\
\hline 14 & $\begin{array}{l}\text { Posterior occipitocervical } \\
\text { decompression/fusion }\end{array}$ & Right upper extremity decreased SSEP & Positioning effect, arm tucked & No deficit \\
\hline 15 & Anterior thoracic osteotomies & $\begin{array}{l}\text { Right lower extremity loss of SSEP } \\
\text { after graft placement }\end{array}$ & None & No deficit \\
\hline 16 & $\begin{array}{l}\text { Posterior thoracolumbar } \\
\text { decompression/fusion TLIF }\end{array}$ & $\begin{array}{l}\text { Right lower extremity loss MEP, } \\
\text { SSEP stable }\end{array}$ & None & No deficit \\
\hline 17 & Anterior cervical corpectomy and fusion & No baseline SSEPS & None & Improved SSEPS \\
\hline 18 & $\begin{array}{l}\text { Posterior thoracolumbar } \\
\text { decompression/fusion PSO }\end{array}$ & $\begin{array}{l}\text { Bilateral lower extremity SSEPS } \\
\text { decreased with rod placement }\end{array}$ & $\begin{array}{l}\text { Rods placed, baseline } \\
\text { SSEPs returned }\end{array}$ & No deficit \\
\hline 19 & T7 spinal cord tumor resection & $\begin{array}{l}\text { Loss of bilateral lower SSEPs } \\
\text { (no MEPs present at baseline) }\end{array}$ & None & $\begin{array}{l}\text { No change from } \\
\text { preoperative function }\end{array}$ \\
\hline 20 & $\begin{array}{l}\text { Posterior then anterior } \\
\text { cervicothoracic fusion }\end{array}$ & Decreased SSEPs post flip & None & No deficit \\
\hline 21 & $\begin{array}{l}\text { Anterior thoracolumbar } \\
\text { decompression/fusion }\end{array}$ & $\begin{array}{l}\text { Thoracotomy, left upper extremity } \\
\text { (down arm) loss SSEPs }\end{array}$ & $\begin{array}{l}\text { Repositioned, large pt, } \\
\text { procedure shortened }\end{array}$ & Transient sensory changes \\
\hline 22 & $\begin{array}{l}\text { Posterior thoracolumbar } \\
\text { decompression/fusion TLIF }\end{array}$ & Right lower extremity loss of MEP & $\begin{array}{l}\text { Needle repositioned, } \\
\text { signals reacquired }\end{array}$ & No deficit \\
\hline 23 & Posterior thoracic fusion & $\begin{array}{l}\text { Bilateral lower extremity loss of MEP } \\
\text { with distraction }\end{array}$ & $\begin{array}{l}\text { Variable signal changes, } \\
\text { returned to baseline }\end{array}$ & No deficit \\
\hline 24 & $\begin{array}{l}\text { Posterior thoracolumbar deformity } \\
\text { correction with fusion }\end{array}$ & $\begin{array}{l}\text { Left lower extremity decreased SSEP, } \\
\text { loss of MEP during correction }\end{array}$ & $\begin{array}{l}\text { Correction held, } \\
\text { increased blood pressure }\end{array}$ & No deficit \\
\hline 25 & $\begin{array}{l}\text { Posterior cervicothoracic } \\
\text { deformity correction with fusion }\end{array}$ & $\begin{array}{l}\text { Bilateral lower extremity loss of MEP, } \\
\text { deacreased SSEPs }\end{array}$ & Correction decreased & No deficit \\
\hline 26 & $\begin{array}{l}\text { Posterior lumbosacral } \\
\text { decompression/fusion }\end{array}$ & Low screw threshould & No breach, screw replaced & No deficit \\
\hline 27 & $\begin{array}{l}\text { Posterior lumbosacral } \\
\text { decompression/fusion TLIF }\end{array}$ & None, spontaneous EMG only & None & Screw in canal \\
\hline 28 & Posterior lumbar decompression/fusion & None, spontaneous EMG only & None & Foot drop, medial left L4screw breach \\
\hline 29 & $\begin{array}{l}\text { Posterior thoracolumbar deformity } \\
\text { correction with fusion }\end{array}$ & Bilateral lower extremity loss of MEP & Decreased correction & No deficit \\
\hline 30 & Anterior cervical corpectomy and fusion & Improved MEP after decompression & None & Improved function \\
\hline 31 & Anterior thoracic discectomy and fusion & Improved MEP after decompression & None & Improved function \\
\hline 32 & Anterior cervical corpectomy and fusion & Improved MEP after decompression & None & Improved function \\
\hline
\end{tabular}




\section{REFERENCES}

1. Modi HN, Suh SW, Yang JH, et al (2009) False-negative transcranial motor-evoked potentials during scoliosis surgery causing paralysis: a case report with literature review. Spine; 34(24):E896-900.

2. Kamerlink JR, Errico T, Xavier S, et al (2010) Major intraoperative neurologic monitoring deficits in consecutive pediatric and adult spinal deformity patients at one institution. Spine; 35(2):240-245.

3. Hart ES, Grottkau BE (2009) Intraoperative neuromonitoring in pediatric spinal deformity surgery. Orthop Nurs; 28; (6):286-292.

4. Pelosi L, Lamb J, Grevitt M, et al (2002) Combined monitoring of motor and somatosensory evoked potentials in orthopaedic spinal surgery. Clin Neurophysiol; 113(7):1082-1091.

5. Noonan KJ, Walker T, Feinberg JR, et al (2002) Factors related to false-verses true-positive neuromonitoring changes in adolescent idiopathic scoliosis surgery. Spine; 27(8):825-830.

6. Langeloo DD, Lelivelt A, Louis Journee $\mathbf{H}$, et al (2003) Transcranial electrical motor-evoked potential monitoring during surgery for spinal deformity; a study of 145 patients. Spine; 28(10):1043-1050.

7. Strahm C, Min K, Boos N, et al (2003) Reliability of perioperative SSEP recordings in spine surgery. Spinal Cord; 41(9): 483-489.

8. Gonzalez AA, Jeyanandarajan D, Hansen C, et al (2009) Intraoperative neurophysiological monitoring during spine surgery: a review. Neurosurg Focus; 27(4):E6.

9. Kim DH, Zaremski J, Kwon B, et al (2007) Risk factors for false positive transcranial motor evoked potential monitoring alerts during surgical treatment of cervical myelopathy. Spine; 32(26):304l-3046.

10. Langeloo DD, Journee HL, de Kleuver $\mathbf{M}$, et al (2007) Criteria for transcranial electrical motor evoked potential monitoring during spinal deformity surgery. A review and discussion of the literature. Neurophysiol Clin; 37(6):431-439.

11. Murkin JM (2004) Perioperative multimodality neuromonitoring: an overview. Semin Cardiothorac Vasc Anesth; 8(2):167-71.

12. Pajewski TN, Arlet V, Phillips LH (2007) Current approach on spinal cord monitoring: the point of view of the neurologist, the anesthesiologist and the spine surgeon. Eur Spine J; 16 Suppl 2:S115-129.

13. Quraishi NA, Lewis SJ, Kelleher MO, et al (2009) Intraoperative multimodality monitoring in adult spinal deformity: analysis of a prospective series of one hundred two cases with independent evaluation. Spine; 34(14):1504-1512. 